\title{
APPROXIMATION OF CAUCHY ADDITIVE MAPPINGS
}

\author{
JaIOK Roh and Hui Joung Shin
}

\begin{abstract}
In this paper, we prove that a function satisfying the following inequality

$\|f(x)+2 f(y)+2 f(z)\| \leq\left\|2 f\left(\frac{x}{2}+y+z\right)\right\|+\epsilon\left(\|x\|^{r} \cdot\|y\|^{r} \cdot\|z\|^{r}\right)$ for all $x, y, z \in X$ and for $\epsilon \geq 0$, is Cauchy additive. Moreover, we will investigate for the stability in Banach spaces.
\end{abstract}

\section{Introduction}

In 1940, S. M. Ulam [12] questioned the following problem : Let $\left(G_{1}, \circ\right)$ be a group and $\left(G_{2}, *\right)$ a metric group with a metric $d(\cdot, \cdot)$. Given $\epsilon>0$, does there exist a $\delta>0$ such that if $f: G_{1} \rightarrow G_{2}$ satisfies

$$
d(f(x \circ y), f(x) * f(y)) \leq \delta \text { for all } x, y \in G_{1},
$$

then there exists a homomorphism $h: G_{1} \rightarrow G_{2}$ with

$$
d(f(x), h(x)) \leq \epsilon \text { for all } x \in G_{1} ?
$$

In 1941, D. H. Hyers [2] proved the following theorem :

Let $E$ and $E^{\prime}$ are Banach spaces. Suppose that a mapping $f: E \rightarrow E^{\prime}$ satisfies

$$
\|f(x+y)-f(x)-f(y)\|_{E^{\prime}} \leq \epsilon
$$

for all $x, y \in E$ and some positive constant $\epsilon$. Then the limit function

$$
L(x)=\lim _{n \rightarrow \infty} \frac{f\left(2^{n} x\right)}{2^{n}}
$$

exists for all $x \in E$ and it is a unique additive mapping satisfying

$$
\|f(x)-L(x)\| \leq \epsilon .
$$

Then, in 1978, Th. M. Rassias [10] proved the following generalization of Hyers [2].

Received April 26, 2007.

2000 Mathematics Subject Classification. Primary: 39B52, 39B62, 39B72.

Key words and phrases. Hyers-Ulam stability, Cauchy additive mapping, Jordan-von Neumann type Cauchy Jensen functional equation. 
Proposition 1.1. Let $f: E \rightarrow E^{\prime}$ be a mapping from a normed vector space $E$ into a Banach space $E^{\prime}$. Suppose that the mapping $f$ satisfies

$$
\|f(x+y)-f(x)-f(y)\| \leq \epsilon\left(\|x\|^{p}+\|y\|^{p}\right)
$$

for all $x, y \in E$ and for $\epsilon>0$ and $p<1$. Then the limit

$$
L(x)=\lim _{n \rightarrow \infty} \frac{f\left(2^{n} x\right)}{2^{n}}
$$

exists for all $x \in E$ and $L: E \rightarrow E^{\prime}$ is the unique additive mapping which satisfies

$$
\|f(x)-L(x)\| \leq \frac{2 \epsilon}{2-2^{p}}\|x\|^{p}
$$

for all $x \in E$. If $p<0$ then inequality (1.1) holds for $x, y \neq 0$ and (1.2) for $x \neq 0$.

In 1991, Z. Gajda [1] provided an affirmative answer to Th. M. Rassias' question whether his theorem can be extended for values of $p$ greater than one. Gajda's proof followed Th. M. Rassias' method described in [10].

However it was shown by Z. Gajda [1] and Th. M. Rassias and P. Semrl [11] that one cannot prove a theorem similar to [10].

In 1982, J. M. Rassias [9] replaced $\|x\|^{p}+\|y\|^{p}$ by $\|x\|^{p} \cdot\|y\|^{q}$ for $p, q \in R$ with $p+q \neq 1$.

More generalizations and applications of the generalized Hyers-Ulam stability to a number of functional equations and mappings can be find in [3], [4], $[5],[6]$ and [7].

C. Park, Y. Cho and M. Han [8] proved that a mapping satisfying one of the following inequalities,

$$
\begin{aligned}
\|f(x)+f(y)+f(z)\| & \leq\left\|2 f\left(\frac{x+y+z}{2}\right)\right\|, \\
\|f(x)+f(y)+f(z)\| & \leq\|f(x+y+z)\|, \\
\|f(x)+f(y)+2 f(z)\| & \leq\left\|2 f\left(\frac{x+y}{2}+z\right)\right\|,
\end{aligned}
$$

is a Cauchy additive mapping. Moreover, they investigated the stability for these mappings in Banach spaces.

In the spirit of Th. M. Rassias' stability approach for approximately homomorphism, many functional inequalities have been studied for decades.

In [8], the first inequality (1.3) had been studied because Jensen function with two variables is difficult to prove additivity, the second inequality (1.4) had been studied because Cauchy function with two variables is also difficult to prove additivity, and the last inequality (1.5) had been studied because it is a generalized Jensen function.

In this paper, we study the inequality,

$$
\|f(x)+2 f(y)+2 f(z)\| \leq\left\|2 f\left(\frac{x}{2}+y+z\right)\right\|
$$


because it is a generalized Cauchy function and is a meaningful inequality to be additive.

We have better results than [8] in the following senses:

(1) We prove that a function satisfying the inequality,

$$
\|f(x)+2 f(y)+2 f(z)\| \leq\left\|2 f\left(\frac{x}{2}+y+z\right)\right\|+\epsilon\left(\|x\|^{r} \cdot\|y\|^{r} \cdot\|z\|^{r}\right)
$$

for all $x, y, z \in X$ and for $\epsilon \geq 0$, is Cauchy additive.

(2) By adapting different method in the proof, we improved the results for the stability of Cauchy additive mappings. They approximated $\frac{f(x)-f(-x)}{2}$ by a Cauchy additive mapping. But we approximate $f(x)$ and $\frac{f(x)-f(-x)}{2}$ by a Cauchy additive mapping.

\section{Main theorem}

Let $X$ be a normed vector space and $Y$ a Banach space. In the following theorem, we prove that a mapping $f: X \rightarrow Y$ satisfying the inequality

$$
\|f(x)+2 f(y)+2 f(z)\| \leq\left\|2 f\left(\frac{x}{2}+y+z\right)\right\|
$$

is Cauchy additive.

Theorem 2.1. Let $f: X \rightarrow Y$ be a mapping such that

$$
\|f(x)+2 f(y)+2 f(z)\| \leq\left\|2 f\left(\frac{x}{2}+y+z\right)\right\|
$$

for all $x, y, z \in X$. Then $f$ is Cauchy additive.

Proof. From (2.1) with $x=y=z=0$, we get $\|5 f(0)\| \leq\|2 f(0)\|$ which implies

$$
\|f(0)\|=0=f(0) .
$$

Also, by letting $x=0$ and $z=-y$ in (2.1) we get

$$
\|f(y)+f(-y)\| \leq\|f(0)\|=0, \quad f(-y)=-f(y) \text { for all } y \in X .
$$

Next, by letting $x=-2 x, y=x$ and $z=0$ in (2.1), we obtain due to (2.3) that

$$
\|f(-2 x)+2 f(x)\|=0, \quad f(x)=2 f\left(\frac{x}{2}\right)=4 f\left(\frac{x}{4}\right)
$$

for all $x \in X$. So, we have due to $(2.2),(2.3)$ and (2.4) that

$$
\begin{aligned}
& \|f(x+y)-f(x)-f(y)\|=\left\|2 f\left(\frac{x+y}{2}\right)+4 f\left(-\frac{x}{4}\right)+4 f\left(-\frac{y}{4}\right)\right\| \\
= & 2\left\|f\left(\frac{x+y}{2}\right)+2 f\left(-\frac{x}{4}\right)+2 f\left(-\frac{y}{4}\right)\right\| \leq 2\left\|2 f\left(\frac{x+y}{4}-\frac{x}{4}-\frac{y}{4}\right)\right\| \\
= & 4\|f(0)\|=0 .
\end{aligned}
$$

Hence, we obtain $f(x+y)=f(x)+f(y)$. 
Next, we will prove that a mapping satisfying the inequality,

$\|f(x)+2 f(y)+2 f(z)\| \leq\left\|2 f\left(\frac{x}{2}+y+z\right)\right\|+\epsilon\left(\|x\|^{r} \cdot\|y\|^{r} \cdot\|z\|^{r}\right)$,

is Cauchy additive.

Theorem 2.2. Let $r>\frac{1}{3}$ and $\epsilon>0$ be real numbers. Suppose that a mapping $f: X \rightarrow Y$ satisfies

(2.5) $\|f(x)+2 f(y)+2 f(z)\| \leq\left\|2 f\left(\frac{x}{2}+y+z\right)\right\|+\epsilon\left(\|x\|^{r} \cdot\|y\|^{r} \cdot\|z\|^{r}\right)$ for all $x, y, z \in X$. Then the function $f$ is a Cauchy additive mapping.

Proof. One can easily get $f(0)=0$ by letting $x=y=z=0$ in (2.5). Also, from (2.5) with $x=2 x, y=-x$ and $z=0$, we get

$$
\|f(2 x)+2 f(-x)\|=0 \text {. }
$$

Next, by letting $x=0$ and $z=-y$ in (2.5), we get

$$
\|f(y)+f(-y)\|=0, \quad f(-y)=-f(y)
$$

for all $y \in X$. Thus, by (2.6) and (2.7) we obtain

$$
f(2 x)=2 f(x), \quad f(x)=2 f\left(\frac{x}{2}\right), \quad f(x)=2^{n} f\left(\frac{x}{2^{n}}\right)
$$

for all $n \in N$ and $f(x)=\lim _{n \rightarrow \infty} 2^{n} f\left(\frac{x}{2^{n}}\right)$ where $x \in X$. Therefore, by (2.5), (2.6) and (2.7) we obtain

$$
\begin{aligned}
&\|f(x)+f(y)-f(x+y)\|=\lim _{n \rightarrow \infty} 2^{n}\left\|f\left(\frac{x}{2^{n}}\right)+f\left(\frac{y}{2^{n}}\right)-f\left(\frac{x+y}{2^{n}}\right)\right\| \\
&= \lim _{n \rightarrow \infty} 2^{n}\left\|f\left(\frac{x}{2^{n}}\right)+f\left(\frac{y}{2^{n}}\right)+f\left(\frac{-x-y}{2^{n}}\right)\right\| \\
& \leq \lim _{n \rightarrow \infty} 2^{n}\left\|f\left(\frac{x}{2^{n}}\right)+f\left(\frac{y}{2^{n}}\right)+\frac{1}{2} f\left(\frac{-x-y}{2^{n-1}}\right)\right\| \\
&+\lim _{n \rightarrow \infty} 2^{n}\left\|\frac{1}{2} f\left(\frac{-x-y}{2^{n-1}}\right)+f\left(\frac{x+y}{2^{n}}\right)\right\| \\
&+\lim _{n \rightarrow \infty} 2^{n}\left\|f\left(\frac{-x-y}{2^{n}}\right)+f\left(\frac{x+y}{2^{n}}\right)\right\| \\
& \leq \lim _{n \rightarrow \infty}\left(\frac{2}{2^{3 r}}\right)^{n} 2^{r-1} \epsilon\|x\|^{r} \cdot\|y\|^{r} \cdot\|x+y\|^{r} \rightarrow 0, \\
& \text { as } n \rightarrow \infty \text { for } r>\frac{1}{3}, \epsilon>0 . \text { Thus } f(x+y)=f(x)+f(y) .
\end{aligned}
$$

Theorem 2.3. Let $r<\frac{1}{3}$ and $f: X \rightarrow Y$ be a mapping satisfying (2.5). Then the function $f$ is a Cauchy additive mapping.

Proof. By the facts,

$$
\|f(2 x)+2 f(-x)\|=0,\|f(x)+f(-x)\|=0, \quad f(-x)=-f(x),
$$

we have

$$
f(x)=\frac{1}{2} f(2 x)=\frac{1}{2^{2}} f\left(2^{2} x\right)=\cdots=\frac{1}{2^{n}} f\left(2^{n} x\right)=\cdots
$$


for all $n \in N$ and $x \in X$. Thus we get $f(x)=\lim _{n \rightarrow \infty} \frac{1}{2^{n}} f\left(2^{n} x\right)$ for all $x \in X$. Hence, similar to the proof of theorem 2.2 , we have

$$
\begin{aligned}
& \|f(x)+f(y)-f(x+y)\|=\lim _{n \rightarrow \infty} \frac{1}{2^{n}}\left\|f\left(2^{n} x\right)+f\left(2^{n} y\right)-f\left(2^{n}(x+y)\right)\right\| \\
\leq & \lim _{n \rightarrow \infty}\left(\frac{2^{3 r}}{2}\right)^{n} 2^{r-1} \epsilon\|x\|^{r} \cdot\|y\|^{r} \cdot\|x+y\|^{r} \rightarrow 0,
\end{aligned}
$$

as $n \rightarrow \infty$, for all $x, y \in X$. Therefore $f(x+y)=f(x)+f(y)$ for all $x, y \in$ $X$.

Next, we will see the stability of the Cauchy additive mappings.

Theorem 2.4. Let $r>1$ and $\epsilon>0$ be real numbers. Suppose that a mapping $f: X \rightarrow Y$ satisfies

(2.9) $\|f(x)+2 f(y)+2 f(z)\| \leq\left\|2 f\left(\frac{x}{2}+y+z\right)\right\|+\epsilon\left(\|x\|^{r}+\|y\|^{r}+\|z\|^{r}\right)$

for all $x, y, z \in X$. Then there exists a unique Cauchy additive mapping $L$ : $X \rightarrow Y$ such that

$$
\|f(x)-L(x)\| \leq \frac{3+2^{r}}{2^{r}-2} \epsilon\|x\|^{r} \quad \text { for all } x \in X .
$$

Moreover, there exists a unique Cauchy additive mapping $S: X \rightarrow Y$ such that

$$
\left\|\frac{f(x)-f(-x)}{2}-S(x)\right\| \leq \frac{1+2^{r}}{2^{r}-2} \epsilon\|x\|^{r} \quad \text { for all } x \in X .
$$

Proof. From (2.9) with $x=y=z=0$, we get $f(0)=0$. Then by letting $z=$ $0, x=-2 x$ and $y=x$ in (2.9), we obtain

$$
\|f(-2 x)+2 f(x)\| \leq\left(1+2^{r}\right) \epsilon\|x\|^{r}
$$

which implies

$$
\left\|f(-x)+2 f\left(\frac{x}{2}\right)\right\| \leq \frac{1+2^{r}}{2^{r}} \epsilon\|x\|^{r} \quad \text { for all } x \in X .
$$

Next, by letting $x=0, y=x$ and $z=-x$ in (2.9), we get

$$
\|f(x)+f(-x)\| \leq \epsilon\|x\|^{r} \quad \text { for all } x \in X .
$$

Hence, we have from (2.13) and (2.14) that

$$
\begin{aligned}
& \left\|2^{l} f\left(\frac{x}{2^{l}}\right)-2^{m} f\left(\frac{x}{2^{m}}\right)\right\| \leq \sum_{j=l}^{m-1}\left\|2^{j} f\left(\frac{x}{2^{j}}\right)-2^{j+1} f\left(\frac{x}{2^{j+1}}\right)\right\| \\
= & \sum_{j=l}^{m-1}\left\|2^{j} f\left(\frac{x}{2^{j}}\right)+2^{j+1} f\left(\frac{-x}{2^{j+1}}\right)-2^{j+1} f\left(\frac{-x}{2^{j+1}}\right)-2^{j+1} f\left(\frac{x}{2^{j+1}}\right)\right\|
\end{aligned}
$$




$$
\begin{aligned}
& \leq \sum_{j=l}^{m-1}\left[\left\|2^{j} f\left(\frac{x}{2^{j}}\right)+2^{j+1} f\left(\frac{-x}{2^{j+1}}\right)\right\|+\left\|2^{j+1} f\left(\frac{-x}{2^{j+1}}\right)+2^{j+1} f\left(\frac{x}{2^{j+1}}\right)\right\|\right] \\
& \leq \frac{3+2^{r}}{2^{r}} \epsilon\|x\|^{r} \sum_{j=l}^{m-1}\left(\frac{2}{2^{r}}\right)^{j}
\end{aligned}
$$

for all nonnegative integers $m$ and $l$ with $m>l$ and all $x \in X$. It means that the sequence $\left\{2^{n} f\left(\frac{x}{2^{n}}\right)\right\}$ is a Cauchy sequence for all $x \in X$. Since $Y$ is complete, the sequence $\left\{2^{n} f\left(\frac{x}{2^{n}}\right)\right\}$ converges. So we can define the mapping $L: X \rightarrow Y$ by $L(x)=\lim _{n \rightarrow \infty} 2^{n} f\left(\frac{x}{2^{n}}\right)$, for all $x \in X$.

Moreover, by letting $l=0$ and passing the limit $m \rightarrow \infty$, we get (2.10).

Next, we claim that $L(x)$ is a Cauchy additive mapping. First of all, we note from (2.14) that

$$
\begin{aligned}
\|L(x)+L(-x)\| & =\lim _{n \rightarrow \infty} 2^{n}\left\|f\left(\frac{x}{2^{n}}\right)+f\left(-\frac{x}{2^{n}}\right)\right\| \leq \lim _{n \rightarrow \infty} 2^{n} \epsilon\left\|\frac{x}{2^{n}}\right\|^{r} \\
& =\lim _{n \rightarrow \infty} \frac{2^{n} \epsilon}{2^{n r}}\|x\|^{r} \rightarrow 0,
\end{aligned}
$$

as $n \rightarrow \infty$ for all $x \in X$. So we have $L(-x)=-L(x)$.

Now, we claim that the mapping $L: X \rightarrow Y$ is a Cauchy additive mapping. We obtain by (2.9), (2.13) and (2.14) that

$$
\begin{aligned}
& \|L(x)+L(y)-L(x+y)\|=\|L(x)+L(y)+L(-x-y)\| \\
= & \lim _{n \rightarrow \infty} 2^{n}\left\|f\left(\frac{x}{2^{n}}\right)+f\left(\frac{y}{2^{n}}\right)+f\left(\frac{-x-y}{2^{n}}\right)\right\| \\
\leq & \lim _{n \rightarrow \infty} 2^{n}\left\|\frac{1}{2} f\left(\frac{-x-y}{2^{n-1}}\right)+f\left(\frac{x}{2^{n}}\right)+f\left(\frac{y}{2^{n}}\right)\right\| \\
& +\lim _{n \rightarrow \infty} 2^{n}\left\|f\left(\frac{-x-y}{2^{n}}\right)+\frac{1}{2} f\left(\frac{x+y}{2^{n-1}}\right)\right\|+\lim _{n \rightarrow \infty} 2^{n-1}\left\|f\left(\frac{-x-y}{2^{n-1}}\right)+f\left(\frac{x+y}{2^{n-1}}\right)\right\| \\
\leq & \lim _{n \rightarrow \infty}\left(\frac{2}{2^{r}}\right)^{n} \frac{\epsilon}{2}\left[2^{r}\|x+y\|^{r}+\|x\|^{r}+\|y\|^{r}\right] \\
& +\lim _{n \rightarrow \infty}\left(\frac{2}{2^{r}}\right)^{n} \epsilon \frac{1+2^{r}}{2}\|x+y\|^{r}+\lim _{n \rightarrow \infty}\left(\frac{2}{2^{r}}\right)^{n-1} \epsilon\|x+y\|^{r} \rightarrow 0,
\end{aligned}
$$

as $n \rightarrow \infty$ for all $x \in X$.

Now, to prove uniqueness of the mapping $L(x)$, let us assume that $T: X \rightarrow$ $Y$ be another Cauchy additive mapping satisfying (2.10).

Then we obtain

$$
\begin{aligned}
& \|L(x)-T(x)\|=2^{n}\left\|L\left(\frac{x}{2^{n}}\right)-T\left(\frac{x}{2^{n}}\right)\right\| \\
\leq & 2^{n}\left[\left\|L\left(\frac{x}{2^{n}}\right)-f\left(\frac{x}{2^{n}}\right)\right\|+\left\|T\left(\frac{x}{2^{n}}\right)-f\left(\frac{x}{2^{n}}\right)\right\|\right] \\
\leq & \left(\frac{2}{2^{r}}\right)^{n}\left(\frac{6+2^{r+1}}{2^{r}-2}\right) \epsilon\|x\|^{r} \rightarrow 0,
\end{aligned}
$$

as $n \rightarrow \infty$ for all $x \in X$. So $L(x)=T(x)$ for all $x \in X$. 
Now, we let $g(x)=\frac{f(x)-f(-x)}{2}$ then

$$
\begin{aligned}
\|2 g(x)-g(2 x)\| & =\left\|f(x)-f(-x)-\frac{f(2 x)-f(-2 x)}{2}\right\| \\
& \leq\left\|f(x)+\frac{f(-2 x)}{2}\right\|+\left\|f(-x)+\frac{f(2 x)}{2}\right\| \\
& \leq\left(1+2^{r}\right) \epsilon\|x\|^{r} \text { for all } x \in X .
\end{aligned}
$$

Hence, we have

$$
\begin{aligned}
\left\|2^{l} g\left(\frac{x}{2^{l}}\right)-2^{m} g\left(\frac{x}{2^{m}}\right)\right\| & \leq \sum_{j=l}^{m-1}\left\|2^{j} g\left(\frac{x}{2^{j}}\right)-2^{j+1} g\left(\frac{x}{2^{j+1}}\right)\right\| \\
& \leq \sum_{j=l}^{m-1}\left(\frac{2}{2^{r}}\right)^{j+1} \frac{\left(1+2^{r}\right) \epsilon}{2}\|x\|^{r}
\end{aligned}
$$

for all nonnegative integers $m$ and $l$ with $m>l$ and all $x \in X$. It means that the sequence $\left\{2^{n} g\left(\frac{x}{2^{n}}\right)\right\}$ is a Cauchy sequence for all $x \in X$. Since $Y$ is complete, the sequence $\left\{2^{n} g\left(\frac{x}{2^{n}}\right)\right\}$ converges. So we can define the mapping $S: X \rightarrow Y$ by $S(x)=\lim _{n \rightarrow \infty} 2^{n} g\left(\frac{x}{2^{n}}\right)$ for all $x \in X$.

Moreover, by letting $l=0$ and passing the limit $m \rightarrow \infty$, we get (2.11).

Next, we claim that $S(x)$ is Cauchy additive. For that we first note $g(-x)$ $=g(-x)$ which means $S(-x)=S(-x)$.

Now, we get by (2.9) and (2.12) that

$$
\begin{aligned}
& \|S(x)+S(y)-S(x+y)\|=\lim _{n \rightarrow \infty} 2^{n}\left\|g\left(\frac{x}{2^{n}}\right)+g\left(\frac{y}{2^{n}}\right)-g\left(\frac{x+y}{2^{n}}\right)\right\| \\
= & \lim _{n \rightarrow \infty} 2^{n}\left\|g\left(\frac{x}{2^{n}}\right)+g\left(\frac{y}{2^{n}}\right)+g\left(\frac{-x-y}{2^{n}}\right)\right\| \\
= & \lim _{n \rightarrow \infty} 2^{n}\left\|\frac{f\left(\frac{x}{2^{n}}\right)-f\left(\frac{-x}{2^{n}}\right)}{2}+\frac{f\left(\frac{y}{2^{n}}\right)-f\left(\frac{-y}{2^{n}}\right)}{2}+\frac{f\left(\frac{-x-y}{2^{n}}\right)-f\left(\frac{x+y}{2^{n}}\right)}{2}\right\| \\
\leq & \lim _{n \rightarrow \infty} 2^{n}\left[\left\|\frac{f\left(\frac{x}{2^{n}}\right)}{2}+\frac{f\left(\frac{y}{2^{n}}\right)}{2}+\frac{f\left(\frac{-x-y}{2^{n-1}}\right)}{4}\right\|+\left\|\frac{f\left(\frac{-x}{2^{n}}\right)}{2}+\frac{f\left(\frac{-y}{2^{n}}\right)}{2}+\frac{f\left(\frac{x+y}{2^{n-1}}\right)}{4}\right\|\right] \\
& +\lim _{n \rightarrow \infty} 2^{n}\left(\left\|\frac{f\left(\frac{-x-y}{2^{n}}\right)}{2}+\frac{f\left(\frac{x+y}{2^{n-1}}\right)}{4}\right\|+\left\|\frac{f\left(\frac{x+y}{2^{n}}\right)}{2}+\frac{f\left(\frac{-x-y}{2^{n}-1}\right)}{4}\right\|\right) \rightarrow 0,
\end{aligned}
$$

as $n \rightarrow \infty$ for all $x, y \in X$. So, we have $S(x+y)=S(x)+S(y)$ for all $x, y \in X$. Assume that $T: X \rightarrow Y$ be another Cauchy additive mapping satisfying (2.11). Then we have

$$
\begin{aligned}
\|S(x)-T(x)\| & =2^{n}\left\|S\left(\frac{x}{2^{n}}\right)-T\left(\frac{x}{2^{n}}\right)\right\| \\
& \leq 2^{n}\left(\left\|S\left(\frac{x}{2^{n}}\right)-g\left(\frac{x}{2^{n}}\right)\right\|+\left\|T\left(\frac{x}{2^{n}}\right)-g\left(\frac{x}{2^{n}}\right)\right\|\right) \\
& \leq\left(\frac{2}{2^{r}}\right)^{n}\left(\frac{1+2^{r}}{2^{r}-2} 2 \epsilon\|x\|^{r}\right) \rightarrow 0,
\end{aligned}
$$


as $n \rightarrow \infty$ for all $x \in X$.

Theorem 2.5. Let $r<1$ and $\epsilon>0$ be real numbers. Suppose that $f: X \rightarrow Y$ be a mapping such that

$$
\|f(x)+2 f(y)+2 f(z)\| \leq\left\|2 f\left(\frac{x}{2}+y+z\right)\right\|+\epsilon\left(\|x\|^{r}+\|y\|^{r}+\|z\|^{r}\right)
$$

for all $x, y, z \in X$. Then there exists a unique Cauchy additive mapping $L$ : $X \rightarrow Y$ such that

$$
\|f(x)-L(x)\| \leq \frac{1+2 \cdot 2^{r}}{2-2^{r}} \epsilon\|x\|^{r} \text { for all } x \in X .
$$

Moreover, there exists a unique Cauchy additive mapping $S: X \rightarrow Y$ such that

$$
\left\|\frac{f(x)-f(-x)}{2}-S(x)\right\| \leq \frac{1+2^{r}}{2-2^{r}} \epsilon\|x\|^{r} .
$$

Proof. Due to (2.12) we get

$$
\left\|f(x)+\frac{1}{2} f(-2 x)\right\| \leq \frac{1+2^{r}}{2} \epsilon\|x\|^{r} \text { for all } x \in X .
$$

Hence, we have by (2.14) and (2.19)

$$
\begin{aligned}
& \left\|\frac{1}{2^{l}} f\left(2^{l} x\right)-\frac{1}{2^{m}} f\left(2^{m} x\right)\right\|=\sum_{j=l}^{m-1}\left\|\frac{1}{2^{j}} f\left(2^{j} x\right)-\frac{1}{2^{j+1}} f\left(2^{j+1} x\right)\right\| \\
\leq & \sum_{j=l}^{m-1}\left[\left\|\frac{1}{2^{j}} f\left(2^{j} x\right)+\frac{1}{2^{j+1}} f\left(-2^{j+1} x\right)\right\|+\frac{1}{2^{j+1}}\left\|f\left(-2^{j+1} x\right)+f\left(2^{j+1} x\right)\right\|\right] \\
\leq & \sum_{j=l}^{m-1}\left[\left(\frac{1+2^{r}}{2^{j+1}}\right) \epsilon\left\|2^{j} x\right\|^{r}+\frac{\epsilon}{2^{j+1}}\left\|2^{j+1} x\right\|^{r}\right] \\
\leq & \sum_{j=l}^{m-1}\left(\frac{1+2 \cdot 2^{r}}{2}\right)\left(\frac{2^{r}}{2}\right)^{j} \epsilon\|x\|^{r}
\end{aligned}
$$

for all nonnegative integers $m$ and $l$ with $m>l$ and all $x \in X$. It means that the sequence $\left\{\frac{1}{2^{n}} f\left(2^{n} x\right)\right\}$ is a Cauchy sequence for all $x \in X$.

Since $Y$ is complete, the sequence $\left\{\frac{1}{2^{n}} f\left(2^{n} x\right)\right\}$ converges. So we can define the mapping $L: X \rightarrow Y$ by $L(x)=\lim _{n \rightarrow \infty} \frac{1}{2^{n}} f\left(2^{n} x\right)$ for all $x \in X$.

Moreover, by letting $l=0$ and passing the limit $m \rightarrow \infty$, we get (2.17).

Now, we claim that the mapping $L: X \rightarrow Y$ is a Cauchy additive mapping. We obtain by $(2.9),(2.19)$ and (2.14) that

$$
\begin{aligned}
& \|L(x)+L(y)-L(x+y)\|=\|L(x)+L(y)+L(-x-y)\| \\
= & \lim _{n \rightarrow \infty} \frac{1}{2^{n}}\left\|f\left(2^{n} x\right)+f\left(2^{n} y\right)+f\left(2^{n+1}(-x-y)\right)\right\|
\end{aligned}
$$




$$
\begin{aligned}
\leq & \lim _{n \rightarrow \infty} \frac{1}{2^{n}}\left\|\frac{1}{2} f\left(2^{n+1}(-x-y)\right)+f\left(2^{n} x\right)+f\left(2^{n} y\right)\right\| \\
& +\lim _{n \rightarrow \infty} \frac{1}{2^{n}}\left\|f\left(2^{n}(-x-y)\right)+\frac{1}{2} f\left(2^{n+1}(x+y)\right)\right\| \\
& +\lim _{n \rightarrow \infty} \frac{1}{2^{n+1}}\left\|f\left(2^{n+1}(-x-y)\right)+f\left(2^{n+1}(x+y)\right)\right\| \\
\leq & \lim _{n \rightarrow \infty}\left(\frac{2^{r}}{2}\right)^{n} \frac{\epsilon}{2}\left[2^{r}\|x+y\|^{r}+\|x\|^{r}+\|y\|^{r}\right] \\
& +\lim _{n \rightarrow \infty}\left(\frac{2^{r}}{2}\right)^{n} \epsilon \frac{1+2^{r}}{2}\|x+y\|^{r}+\lim _{n \rightarrow \infty}\left(\frac{2^{r}}{2}\right)^{n+1} \epsilon\|x+y\|^{r} \rightarrow 0,
\end{aligned}
$$

as $n \rightarrow \infty$ for all $x \in X$. The rest of the proof is similar with Theorem 2.4.

We note from (2.15) that

$$
\left\|g(x)-\frac{g(2 x)}{2}\right\| \leq \frac{\left(1+2^{r}\right) \epsilon}{2}\|x\|^{r} \quad \text { for all } x \in X .
$$

Hence, we get

$$
\begin{aligned}
\left\|\frac{1}{2^{l}} g\left(2^{l} x\right)-\frac{1}{2^{m}} g\left(2^{m} x\right)\right\| & \leq \sum_{j=l}^{m-1}\left\|\frac{1}{2^{j}} g\left(2^{j} x\right)-\frac{1}{2^{j+1}} g\left(2^{j+1} x\right)\right\| \\
& \leq \sum_{j=l}^{m-1}\left(\frac{2^{r}}{2}\right)^{j} \frac{\left(1+2^{r}\right) \epsilon}{2}\|x\|^{r}
\end{aligned}
$$

for all nonnegative integers $m$ and $l$ with $m>l$ and all $x \in X$. It means that the sequence $\left\{\frac{1}{2^{n}} g\left(2^{n} x\right)\right\}$ is a Cauchy sequence for all $x \in X$. Since $Y$ is complete, the sequence $\left\{\frac{1}{2^{n}} g\left(2^{n} x\right)\right\}$ converges. So we can define the mapping $S: X \rightarrow Y$ by $S(x)=\lim _{n \rightarrow \infty} \frac{1}{2^{n}} g\left(2^{n} x\right)$, for all $x \in X$.

Moreover, by letting $l=0$ and passing the limit $m \rightarrow \infty$, we get (2.18). The rest of the proof is similar with Theorem 2.4.

\section{References}

[1] Z. Gajda, On stability of additive mappings, Internat. J. Math. Math. Sci. 14 (1991), no. $3,431-434$.

[2] D. H. Hyers, On the stability of the linear functional equation, Proc. Nat. Acad. Sci. U. S. A. 27 (1941), 222-224.

[3] D. H. Hyers, G. Isac, and Th. M. Rassias, Stability of functional equations in several variables, Progress in Nonlinear Differential Equations and their Applications, 34. Birkhauser Boston, Inc., Boston, MA, 1998.

[4] K. Jun and Y. Lee, A generalization of the Hyers-Ulam-Rassias stability of the Pexiderized quadratic equations, J. Math. Anal. Appl. 297 (2004), no. 1, 70-86.

[5] S. M. Jung, Hyers-Ulam-Rassias stability of functional equations in mathematical analysis, Hadronic Press, Inc., Palm Harbor, FL, 2001.

[6] C. Park, Homomorphisms between Poisson JC*-algebras, Bull. Braz. Math. Soc. (N.S.) 36 (2005), no. 1, 79-97.

[7] — Hyers-Ulam-Rassias stability of homomorphisms in quasi-Banach algebras, Bull. Sci. Math. (to appear). 
[8] C. Park, Y. Cho, and M. Han, Functional inequalities associated with Jordan-von Neumann-type additive functional equations, J. Inequal. Appl. (2007), 1-13.

[9] J. M. Rassias, On approximation of approximately linear mappings by linear mappings, J. Funct. Anal. 46 (1982), no. 1, 126-130.

[10] Th. M. Rassias, On the stability of the linear mapping in Banach spaces, Proc. Amer. Math. Soc. 72 (1978), no. 2, 297-300.

[11] Th. M. Rassias and P. Semrl, On the behavior of mappings which do not satisfy HyersUlam stability, Proc. Amer. Math. Soc. 114 (1992), no. 4, 989-993.

[12] S. M. Ulam, A collection of mathematical problems, Interscience Tracts in Pure and Applied Mathematics, no. 8 Interscience Publishers, New York-London 1960.

JAIOK ROH

DEPARTMENT OF MATHEMATICS

HALLYM UNIVERSITY

Chuncheon, Korea

E-mail address: joroh@dreamwiz.com

Hui Joung SHIN

DePaRTMENT OF MATHEMATICS

Chungnam National University

DAEJEON, KoREA

E-mail address: coolguy414@hanmail.net 